\title{
Design of Prediction System for Key Performance Indicators in Balanced Scorecard
}

\author{
Ahmed Mohamed \\ Abd El-Mongy. \\ Faculty of Systems and \\ Computers Engineering, \\ Al-Azhar University Cairo, \\ Egypt.
}

\author{
Alaa el-Deen \\ Hamouda. \\ Faculty of Systems and \\ Computers Engineering, \\ Al-Azhar University, Cairo, \\ Egypt.
}

\author{
Nihal Nounou. \\ Adjunct Prof at Faculty of \\ Computer and Information \\ Science, Arab Academy \\ for Science and \\ Technology and Maritime \\ Transport, Cairo, Egypt.
}

\author{
Abdel-Moneim A. \\ Wahdan. \\ Faculty of Engineering \\ Ain-Shams University \\ Cairo, Egypt.
}

\begin{abstract}
The balanced scorecard (BSC) is a performance management system that supplements traditional financial measures with the criteria that measure the performance from different perspectives. For strategists, they need to predict the KPIs future values to make good decisions during the design of BSC and the determination of the suitable target for each objective and KPI. From historical data, the dependency between KPIs can be discovered through developing traditional prediction model. Hence, the KPI future values can be predicted. However, such prediction does not consider the nature of KPIs in the BSC. The historical values of KPIs depend on the previously settled targets for objectives and KPIs. This raises the challenge of finding a solution to make more accurate prediction that considers the real values of KPIs beside the previous settled targets. For achieving that, we propose a solution that uses fuzzy logic to categorize the KPI values and then predict the future KPI values. Then, we develop a third predictor model as a data fusion module to predict the KPI values depending on both previous values and category predictors. We find that the prediction accuracy of our proposed solution significantly overcomes the normal values prediction of KPIs.
\end{abstract}

\section{Keywords}

Data Mining, Balanced Scorecard, Key Performance Indicators, Association Rule, Fuzzy, Data Fusion, Decision Tree and KPI Prediction.

\section{INTRODUCTION}

The Balanced Scorecard (BSC), as documented by Kaplan and Norton, describes a methodology used for measuring success and setting goals from financial and operational viewpoints [1]. With these measures, leaders can manage their strategic vision and adjust it for change. The balanced scorecard links performance measures by looking at a business's strategic vision from four different perspectives: Financial, Customer, Learning and Internal Business Processes. Each of the four perspectives is considered under various parameters. These parameters include goals that have to be achieved in order to become successful. Key performance indicators (KPIs) are parameters that will be used to know if success is achieved; and targets are quantitative value that will be used to determine the success of the KPI.

The task to set target is very hard and the result of target-setting in the workplace seems unable to reach satisfaction. Setting targets can be fraught with many problems. For example, if set is too high, targets create stress and de-motivation; if set is too low, targets encourage complacency. The history values may play important role to set target correctly [2].

A lot of researches have carried out involving the application of the BSC methodology in several business domains [3-6]. Shana and Venkatachalam [7] identify Key Performance Indicators and predict the result from Student Data. However, to our knowledge, no research has applied it to predict key performance indicators in balanced scorecard considering the relations between strategic objects.

The relation between strategic objects are called Cause and Effect relation. The Cause and Effect relation exists between objective and other objectives or between KPI and other KPIs. Accurate predictions support us to develop System for target setting depending on Cause and Effect relationship analysis and history target setting data.

We propose a solution that uses Association Rules to discover the relations between KPIs. Then, we use the discovered relations and KPIs history of values as input to Fuzzy Logic Component to predict the KPI values. In parallel, we use another method that depends on neural network to predict the KPI values. Then, as data fusion we tune the output results using decision tree to get more accurate predictions. By that, we get more accurate predictions and hence we get better decisions about targets. By following these steps, if we have five KPIs and we know four KPIs values we can predict fifth KPI value more accurately.

This paper is organized as follows: Section 1 is the introduction. Section 2 is an overview of predication Algorithms. Section 3 is the description of the proposed approach. Section 4 is the implementation of the proposed approach. Section 5 is the evaluation. Section 6 is the conclusion.

\section{OVERVIEW OF PREDICTION ALGORITHMS}

An overview about the used algorithms is briefly described briefly as follows.

\subsection{Association Rules}

Data Mining is automated process of finding relationships and patterns in the stored data. One of the data mining output is associating what events are likely to occur together. Association rules mining is one of the major techniques of data mining and it is perhaps the most common form of local-pattern discovery in the unsupervised learning systems [8].

The Apriori Algorithm can be used to generate all frequent itemset. The Frequent itemset the one whose support is greater than some user-specified minimum support (denoted Lk, where $\mathrm{k}$ is the size of the itemset). The Candidate itemset is the 
potentially frequent one (denoted $\mathrm{Ck}$, where $\mathrm{k}$ is the size of the itemset).

\subsection{Fuzzy logic}

Fuzzy Logic was initiated in 1965 [9] by Lotfi A. Zadeh , professor for computer science at the University of California in Berkeley. Basically, Fuzzy Logic is a multivalued logic that allows intermediate values to be defined between conventional evaluations like true/false, yes/no, high/low, etc. Notions like rather tall or very fast can be formulated mathematically and processed by computers, in order to apply a more human-like way of thinking in the programming of computers.

The fuzzy rules and the combination of the results of the individual rules are performed using fuzzy set operations as in table 1.

\section{Table 1, Fuzzy Set Operations}

\begin{tabular}{|l|l|}
\hline And method & Min \\
\hline Or method & Max \\
\hline Implication & Min \\
\hline Aggregation & Max \\
\hline Defuzzification & Centroid \\
\hline
\end{tabular}

\subsection{Artificial Neural Network:-}

The artificial neural network (ANN), often just called a "neural network" (NN), is a mathematical model or computational model based on biological neural networks. In other words, it is an emulation of biological neural system. It consists of an interconnected group of artificial neurons and processes information using a connectionist approach to computation. In most cases, an ANN is an adaptive system that changes its structure based on external or internal information that flows through the network during the learning phase [10].

\subsection{Decision Tree}

Decision trees are built of nodes, branches and leaves that indicate the variables, conditions, and outcomes, respectively. The most predictive variable is placed at the top node of the tree. The operation of decision trees is based on the C4.5 algorithms. C4.5 adopts greedy approach in which decision trees are constructed in a top- down recursive divide and conquer manner.

\section{PROPOSED APPROACH}

It is required to predict KPI values depending on relations between KPIs inside BSC. We propose an approach illustrated as in figure 1. In the first phase, we gather all the relations between KPIs using association rules. On the one hand, we use these rules with Fuzzy to predict KPI values. In parallel, we predict KPIs values by Neural Network. After that, we fuse the prediction results using C4.5 Decision tree.

As shown in figure 1, our proposed solution includes the following components:

\subsection{Discovery of Association Rules}

\subsubsection{Define Status}

Status is an indication, which shows the region that the KPI falls in. As in Table 2, Alarm is a KPI's warning value. Target is the level of performance or rate of improvement required for a particular KPI. Stretch Target is an extreme KPI accomplishment that must be marked and analyzed as being either exceptional performance or a case that requires further investigation. The status falls in one of the regions shown in table 2 .
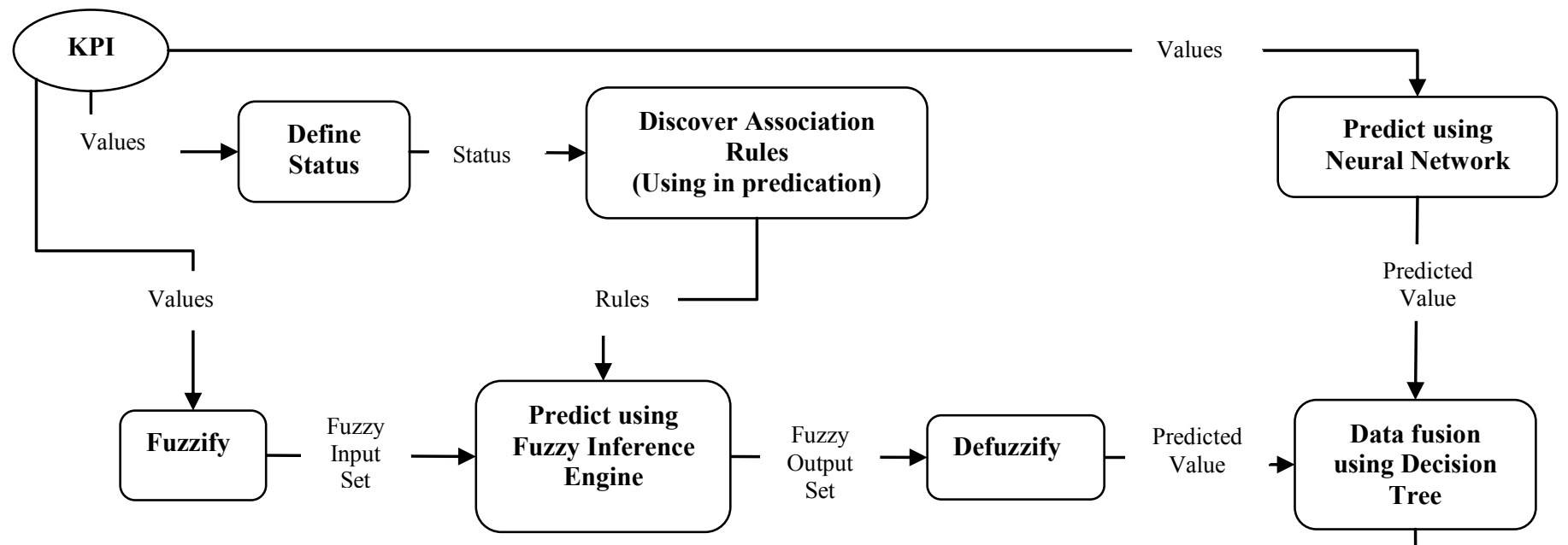

Predict using Neural Network

Fig.1 is a Block Diagram for proposed approach. 1 apie $\angle$, status veimitions

\begin{tabular}{|l|l|}
\hline Red & In the alarm zone \\
\hline
\end{tabular}




\begin{tabular}{|l|l|}
\hline Yellow & Between alarm and target \\
\hline Green & Equal or Exceed target \\
\hline Blue & Equal or Exceed Stretched target \\
\hline
\end{tabular}

In this phase, we translate the value to its related status. It is important to note that the targets and alarms can be changed from a year to another and hence all statuses will differ upon that. So, the value of a KPI can be interpreted into different statuses from a year to another according to the settled targets and alarms. Inputs to this phase are values. Outputs are Status.

\subsubsection{Discover Association Rules}

We use Apriori association rules algorithm to predict KPI status. The Apriori Algorithm can be used to generate all frequent itemset. Therefore, we can predict all the KPIs status. As for the business rule in strategy maps the objective and KPIs in a perspective can be affected by the objectives and KPIs in the lower perspectives, not in the higher. The inputs to this phase are status, the outputs are rules.

\subsection{Prediction Using Fuzzy Logic 3.2.1 Fuzzify}

The process of fuzzy logic is a crisp set of input data KPIs values that are gathered and converted to a fuzzy set using fuzzy linguistic variables, fuzzy linguistic terms and membership functions. Inputs to this phase are the values. Outputs are the fuzzy inputs set.

\subsubsection{Predict Using Fuzzy Inference Engine}

The output rules from discover Association are used as rules to Fuzzy logic. It is necessary to construct the membership functions. We design them as shown in figure 2. Each range has known boundaries so there are no inferences between these ranges. On the one hand, the Red is drawn as a triangular. When the Red decreases, this value goes away from the alarm zone. The yellow also is drawn as a triangular. When the yellow decreases, this value goes away from the target zone. On the other hand, the Green zone is drawn as a triangular. When the Green increases, the value gets close to the target zone. The Blue is drawn as a triangular. When the blue increases, the value gets close to the Stretch target zone.

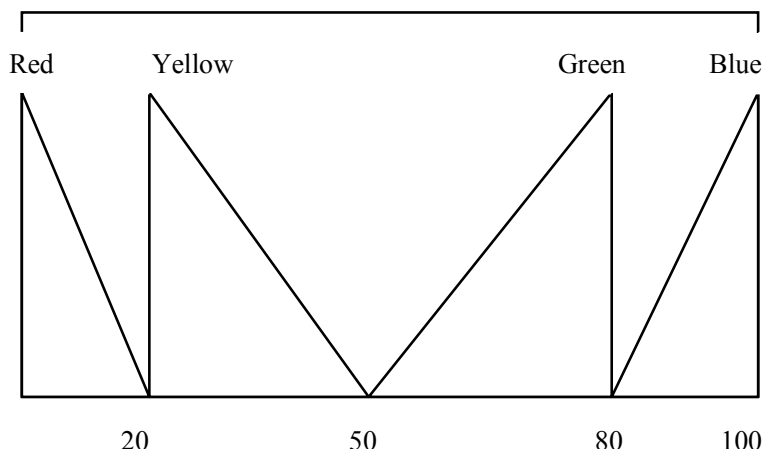

Fig.2 Define type for Membership Functions.

We convert the association rules to fuzzy rules. Rule base is constructed to control the output variable. Inputs to this phase are fuzzy inputs set and Rules, Outputs are fuzzy outputs set.

\subsubsection{Defuzzify}

After the inference step, the overall result is a fuzzy value. This result should be defuzzified to obtain a final output. Defuzzification is performed according to the membership function of the output variable. The inputs to this phase are the fuzzy outputs set; the outputs are the predicted values.

\subsection{Prediction Using Neural Network}

In this phase, we discover how KPI shows tomorrow. We use neural networks. Such prediction does not consider the nature of KPI in the BSC from values and status. It depends on KPI values only and neglect KPI status. In that way, Prediction using Neural Network loses the benefit in the BSC business meaning and it gets predication from only one perspective. Hence, in this phase considering the nature of KPI in the BSC from values is our target. Inputs to this phase are values, Outputs are Predicted values.

\subsection{Data fusion using decision tree,}

We enhance the output by taking the output from normal prediction (neural network) result and fuzzy result, then fusing them using $\mathrm{C} 4.5$ decision tree. The inputs to this phase are the predicted values from both Neural Network Module and Fuzzy Logic Module. The outputs are the fused results that should be the most accurate prediction.

\section{IMPLEMENTATION}

We have data set for a company in Egypt that used BSC framework decade ago. We implement our approach at Corporate Balanced Scorecard as in figure 3. It has more than 532 observations values for different KPIs. It is used to review and monitor the corporate objectives and KPIs. This BSC contains four perspectives. Finance perspective is the highest priority one that answers question on how the firm looks to shareholders. Customer perspective answers question on how customers see the firm. Operations Perspective answers question how well it manages its operational processes. Learning and Growth perspective answers question if the firm can continue to improve and create value.

Fig.3, Corporate Balanced Scorecard

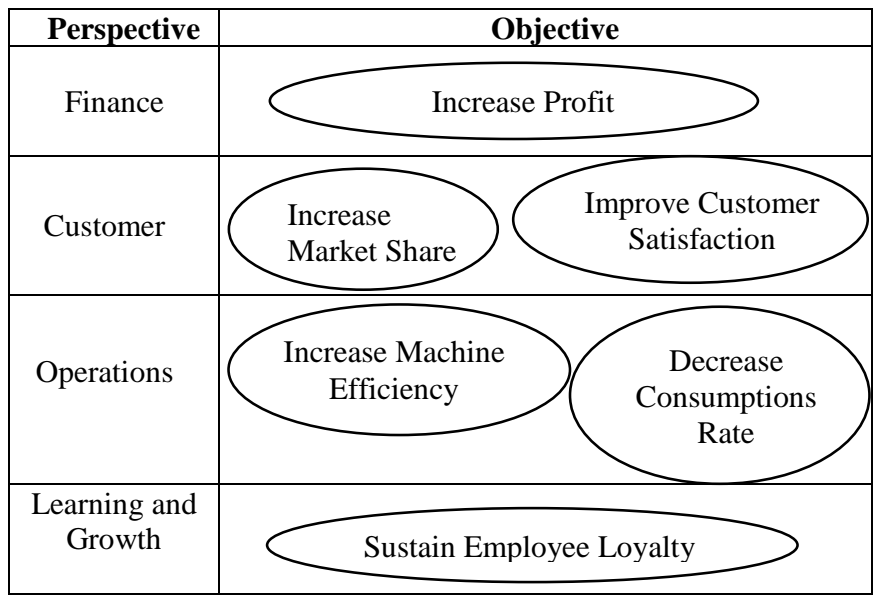

The Corporate Balanced Scorecard depends on several perspectives. Every perspective includes several objectives. For example "Finance" perspective includes "Increase profit objective". From Customer perspective the objective is to "Increase market share" and "Improve customer satisfaction". From Operations perspective it is required "Increase Machine Efficiency" and "Decrease Consumptions Rate". Learning and 
Growth Perspective includes "Sustain Employee loyalty" objective.

These objectives are measured by several KPIs as in table 4:

- Monthly Shipping "Ton" compared to same month last year.

This KPI is a measure for "Increase profit" objective. It shows how many tons are produced compared to the same month last year.

- Number of New Customers.

This KPI is a measure for "Increase Market Share" objective. It shows the Number of New Customers.

- \% of Orders Delivered on-time.

This KPI is a measure for "Improve Customer Satisfaction" objective. It shows the percentage of Orders Delivered ontime.

- \% Total Plant Waste.

This KPI is a measure for "Increase Machine Efficiency" objective. It shows percentage of Total Plant Waste in the manufactory process.

- Average Material Consumption "Kg/Ton produced". This KPI is a measure for "Decrease Consumptions rate" Objective. It shows Average Material Consumption "Kg/Ton produced".

- Number of Employees Leaving.

This KPI is a measure for "Learning and Growth" Objective. It shows the loyalty of employees through measuring the number of employees leaving yearly.

Table 4, the KPIs of Objectives

\begin{tabular}{|c|c|}
\hline Objective & KPI \\
\hline Increase Profit & $\begin{array}{c}\text { Monthly Shipping "Ton" compared to } \\
\text { same month last year }\end{array}$ \\
\hline Increase Market Share & Number of New Customers \\
\hline $\begin{array}{c}\text { Improve Customer } \\
\text { Satisfaction }\end{array}$ & $\%$ of Orders Delivered on-time \\
\hline $\begin{array}{c}\text { Increase Machine } \\
\text { Efficiency }\end{array}$ & $\%$ Total Plant Waste \\
\hline $\begin{array}{c}\text { Decrease } \\
\text { Consumptions Rate }\end{array}$ & $\begin{array}{c}\text { Average Material Consumption } \\
\text { "Kg/Ton produced" }\end{array}$ \\
\hline Learning and Growth & Number Of Employees Leaving \\
\hline
\end{tabular}

Our proposed system includes the following components:

\subsection{Discovery of Association Rules.}

We run association rule Apriori Algorithm to discover the relations between KPIs. Apriori Algorithm uses status as Inputs. Below are samples of discovered rules:-

- IF "Number of New Customers" is in the Red Zone Then the " Monthly Shipping Ton compared to same month last year" is in the Red Zone.

- IF "Number of New Customers" is in the Blue Zone Then the "Monthly Shipping Ton compared to same month last year" is in the Blue Zone.

- IF "\% of Orders Delivered on-time" is in the Blue Zone and "\% Total Plant Waste" is in the Yellow Zone Then the "Monthly Shipping "Ton" compared to same month last year " is in the Blue Zone.

- IF "Average Material Consumption "Kg/Ton produced" is in the Red Zone and "Number of New Customers" is in the Yellow Zone Then the "Monthly Shipping "Ton" compared to same month last year" is in the Red Zone.
From these rules the relations between objectives can be discovered and the strategy map can be redrawn as figure 4 . "Increase market share" Objective, "Improve customer satisfaction" Objective and "Increase Machine Efficiency" Objective lead to "Increase profit" objective. Also "Increase Machine Efficiency" and "Decrease Consumptions Rate" are lead to "Improve customer satisfaction" Objective. "Sustain Employee Loyalty" Objective supports "Decrease Consumptions Rate" Objective.

Fig.4, Corporate Balanced Scorecard

\begin{tabular}{|c|c|c|}
\hline Perspective & Objective \\
\hline Finance & Increase Profit \\
\hline Operations & Increase \\
\hline $\begin{array}{c}\text { Learket Share } \\
\text { Growth }\end{array}$ & Sustain Employee Loyalty \\
\hline
\end{tabular}

\subsection{Prediction using Fuzzy}

Now we have all the relations that discovered by association rules algorithm. We can use these relations as input rules to Fuzzy function. We have all KPIs status like Red, Yellow, Green and Blue. Also we have all KPIs values, so we can use both status and values as Inputs to fuzzy. For example to predict "Monthly Shipping Ton compared to same month last year" KPI we use the values for other KPIs after Fuzzification of them as Inputs to the rules Illustrated in the section 4.1.

\subsection{Prediction using Neural Network}

We make value based predication using artificial neural network. Figure 5 shows Neural Network with four inputs and one hidden layer, which contains two nodes and one output. Predication using Neural Network has KPIs values as input and the predicated KPI value as output.

\subsection{Data Fusion}

The results (predicted values) from Neural Network module and Fuzzy Module are fed to decision tree to generate the most accurate predication values as output. 


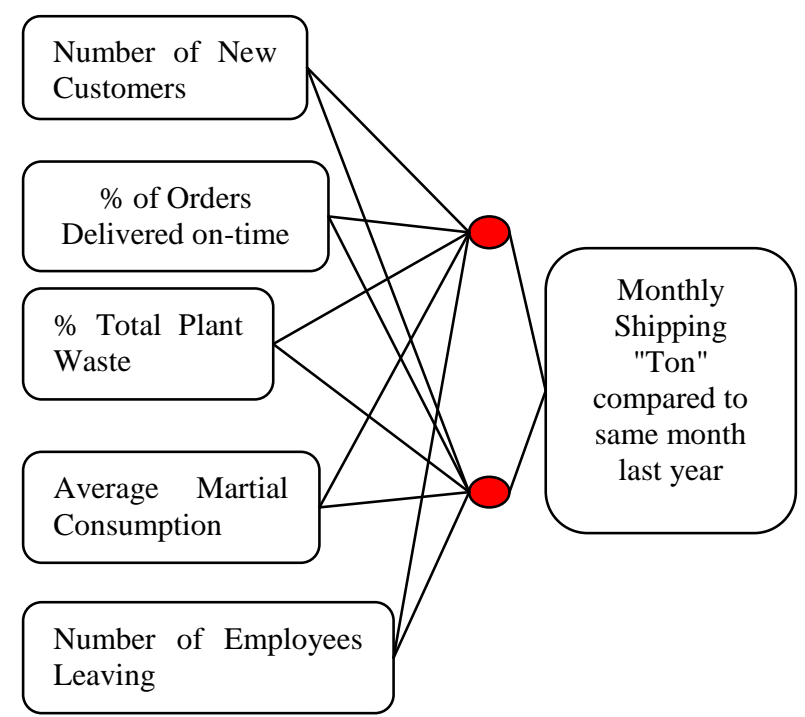

Fig.5 KPIs with Neural network

\section{EVALUATION}

To study the effect of the proposed solution, we have a lot of KPIs and observation values. We repeat the above steps to predict others KPIs like the "Number of the New Customers". Input KPIs are \% of Orders Delivered on-time, \% Total Plant Waste, Average Material Consumption "Kg/Ton produced", and the Number of Employees Leaving. We apply these scenarios on 25 BSCs. Every BSC contains from four to six objectives and KPIs on average.

We use the rules discovered by the association rule as input to Fuzzy Algorithm then predict the values. Figure 6 displays the results. It shows that the average error of the prediction using fuzzy is $6.3 \%$. On the other hand the average error using Neural Network is $5.2 \%$. Finally the average error for the overall proposed system is $1.5 \%$. It is evident that our proposed system decreases the error significantly and improves the prediction accuracy.

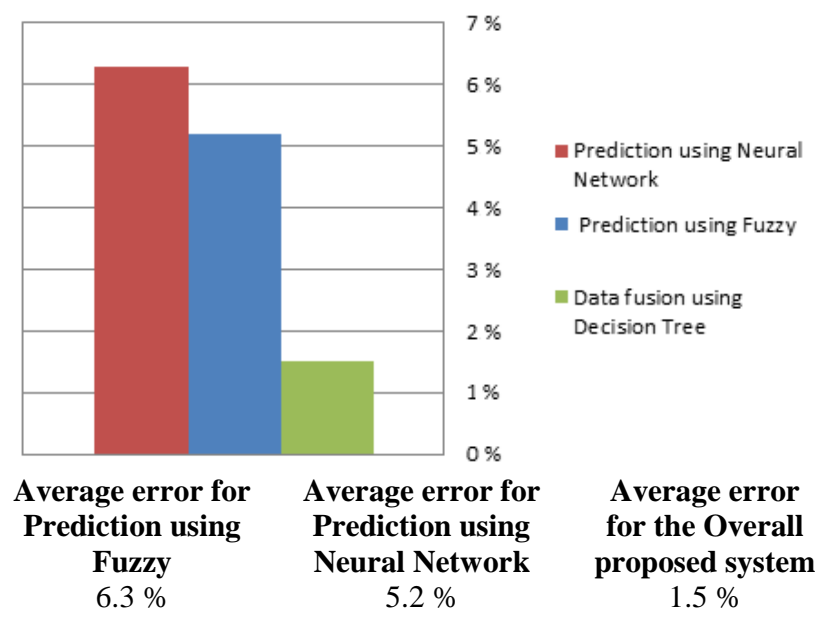

Fig 6: Comparing Average errors in all steps

\section{CONCLUSIONS}

When we have a good predication system, it will be useful to help in the strategic management process. The proposed solution exploited the characteristics of BSC to develop integrated solution to find an accurate prediction for the KPIs. We propose a system composed of several modules. The Association rule and Fuzzy logic modules have an average error of $6.3 \%$. When Neural Network module is used as value based prediction, the average error is $5.2 \%$. The total average error became $1.5 \%$ after using data fusion to tune the output from both Fuzzy and Neural network Components.

\section{REFERENCES}

[1] Leen Yu M and et al. 2009. The e-balanced scorecard (eBSC) for measuring academic staff performance excellence. Journal of Higher Education 2009; 57(6):813828.

[2] Alan Meekings, Steve Briault and Andy Neely. 2011. How to avoid the problems of target-setting. Measuring Business Excellence, Vol. 15 Iss: 3, pp.86 - 98.

[3] Gomes R. 2009 The Balanced Scorecard as a Performance Management Tool for Third Sector Organizations: the Case of

[4] the Arthur Bernardes Foundation, Brazil. Brazilian Administration Review, Curitiba; 6(4), art. 5:354-366.

[5] Zhang T and Gao L. 2008. Study on the Application of Dynamic Balanced Scorecard in the Service Industry. IEEE

[6] International Conference on Intelligent Computation Technology and Automation; 1:1158-1162. (20).

[7] Amaratunga D and et al.2002 Application of the balanced score-card to develop a conceptual framework to measure facilities management performance within NHS facilities. International Journal of Health Care Quality Assurance; 15(4):141-151.

[8] J. Shana and T. Venkatachalam. July 2011, Identifying Key Performance Indicators and Predicting the Result from Student Data, International Journal of Computer Applications (0975 - 8887) Volume 25- No.9.

[9] Alaa Hamouda, "BSCBAS. 2012 A Balanced Scorecard Based Appraisal System for improving software organizations performance". Journal of Software Maintenance and Evolution: Research and Practice, Willy Publisher, 30 JUL 2012. DOI: 10.1002/smr1566.

[10] Sanjeev Rao and Priyanka Gupta. JAN - March 2012. Implementing Improved Algorithm Over APRIORI Data Mining Association Rule Algorithm. INTERNATIONAL JOURNAL OF COMPUTER SCIENCE and TECHNOLOGY (IJCST), V 3.

[11] M. Hellmann. March 2001. Fuzzy Logic Introduction .

[12] YASHPAL SINGH and ALOK SINGH CHAUHAN. 2005 - 2009. Journal of Theoretical and Applied Information Technology.

[13] ]Gaurav L. Agrawal and Prof. Hitesh Gupta, Optimization of C4.5 Decision Tree Algorithm for Data Mining Application; March 2013; International Journal of Emerging Technology and Advanced Engineering; Volume 3, Issue 3, March 2013. 